BULLETIN OF THE

AMERICAN MATHEMATICAL SOCIETY

Volume 79, Number 4, July 1973

\title{
THE VITALI-HAHN-SAKS AND NIKODYM THEOREMS FOR ADDITIVE FUNCTIONS. II
}

\author{
BY R. B. DARST \\ Communicated by Robert Bartle, January 10, 1973
}

\begin{abstract}
In this note appropriate versions of the Vitali-Hahn-Saks and Nikodym theorems are established for $s$-bounded additive set functions with values in an abelian topological group $G$.
\end{abstract}

Although we shall use + and 0 to denote addition and identity in both $G$ and $R$, the real numbers, no confusion should arise. Thus we denote by $\mathscr{U}$ the set of symmetric neighborhoods of 0 in $G$, and for $U \in \mathscr{U}$ we set $1 U=U$ and $(n+1) U=\{x+y: x \in n U, y \in U\}, n \in N$, the set of positive integers.

A subset $H$ of $G$ is said to be bounded if for each $U \in \mathscr{U}$ there exists $n \in N$ such that $H \subset n U$.

We suppose that finite subsets of $G$ are bounded.

Let $\Omega$ be a nonempty set and let $\mathscr{S}$ be a sigma algebra of subsets of $\Omega$.

A function $\mu$ from $\mathscr{S}$ to $G$ is said to be additive if $\mu(\phi)=0$ and $\mu(E \cup F)+\mu(E \cap F)=\mu(E)+\mu(F), E, F \in \mathscr{S}$.

An additive function $\mu$ is said to be $s$-bounded (cf. [1],[5]) if $\lim _{n} \mu\left(E_{n}\right)$ $=0$ (i.e., for each $U \in \mathscr{U}$ there is $m \in N$ such that $\mu\left(E_{n}\right) \in U, n>m$ ) for each sequence $\left\{E_{n}\right\}$ of pairwise disjoint elements of $\mathscr{S}$.

Notice that if $\mu$ is $s$-bounded, $U \in \mathscr{U}$, and $\left\{E_{n}\right\}$ is a sequence of pairwise disjoint elements of $\mathscr{S}$, then there exists $n \in N$ such that if $M$ is a finite subset of $N^{n}=\{k \in N: k \geqq n\}$ then $\left(\sum_{i \in M} \mu\left(E_{i}\right)\right) \in U$.

An additive function $\mu$ is said to be bounded if $\mu(\mathscr{S})=\{\mu(E): E \in \mathscr{S}\}$ is a bounded subset of $G$.

For the case when $\mu$ is sigma-additive, versions of our results can be found in [4]; for the case where $\mu$ is merely additive and $G=R$, one can refer to $[2]$.

Our version of Nikodym's theorem, a striking improvement of the principle of uniform boundedness, follows.

THEOREM 1. Suppose that $T$ is a set of s-bounded functions such that for each $E \in \mathscr{S}$ the set $T(E)=\{\mu(E): \mu \in T\}$ is bounded, then $T(\mathscr{S})=\{\mu(E): \mu \in T$, $E \in \mathscr{S}\}$ is bounded.

AMS (MOS) subject classifications (1970). Primary 28-00, 28A45, 46G99.

Key words and phrases. Vitali-Hahn-Saks theorem, Nikodym theorem, s-bounded, additive set function, abelian group. 
ProOF. It suffices to consider the case where $T$ is a countable set $\left\{\mu_{k}\right\}$. Suppose on the contrary that $T(\mathscr{S})$ is not bounded; then let $U \in \mathscr{U}$ such that $T(\mathscr{S}) \notin n U, n \in N$. Since $T(\Omega)$ is bounded, there is $q_{0} \in N$ such that $T(\Omega) \subset q_{0} U$. Notice that if $\mu_{k_{1}}(E) \notin\left(2 q_{0}+p_{1}\right) U$, then $\mu_{k_{1}}(\Omega-E) \notin$ $\left(q_{0}+p_{1}\right) U$; choose such $k_{1}$ for $p_{1}=2$. At least one of the restrictions to $E$ and $(\Omega-E)$ behaves like the original problem. Thus, we set $\Omega_{1}=E$, $\mathscr{S}_{1}=\left\{E \cap \Omega_{1}: E \in \mathscr{S}\right\}, T_{1}=\left\{\mu \mid \Omega_{1}: \mu \in T\right\}$, and $F_{1}=\Omega-E$, if $T_{1}\left(\mathscr{S}_{1}\right)$ is unbounded; otherwise, $\Omega_{1}=\Omega-E, \cdots$. Iterating this process and relabeling if necessary $\left(k_{j} \rightarrow j\right)$ we obtain a sequence $\left\{F_{j}\right\}$ of pairwise disjoint elements of $\mathscr{S}$ such that $\mu_{k}\left(F_{k}\right) \in\left\{q_{k} U-\left[\left(\sum_{j<k} q_{j}\right)+k+1\right] U\right\}$, $k \in N$. Partitioning $\left\{F_{k}\right\}_{k \geqq 2}$ into a sequence of subsequences and using the fact that $\mu_{1}$ is $s$-bounded yields a subsequence $\left\{F_{n_{i}}\right\}_{i \geqq 1}$ such that $\mu_{1}\left(\mathscr{S} \cap\left(\bigcup_{i \geqq 1} F_{n_{i}}\right)\right) \subset U$. Repeating this process gives us a subsequence $k_{1}=1, k_{2}=n_{1}, \cdots$ such that $\mu_{k_{j}}\left(\mathscr{S} \cap\left(\bigcup_{i>j} F_{k_{i}}\right)\right) \subset U, j \in N$. Set $G=$ $\bigcup_{i} F_{k_{i}}$ and notice that the contradiction $\mu_{k_{i}}(G) \notin k_{i} U, i \in N$, follows. Thus Theorem 1 is established.

When $T$ is a one element set, Theorem 1 specializes as follows.

Corollary 1. An s-bounded function is bounded.

Theorem 1 also permits us to assert that if $\mu_{k}(E)$ is Cauchy, $E \in \mathscr{S}$, and $G$ is complete, then the additive function $\mu$ defined by $\mu(E)=\lim \mu_{k}(E)$ is bounded; a corollary of the following Vitali-Hahn-Saks theorem asserts that $\mu$ is $s$-bounded.

A sequence $\left\{\mu_{k}\right\}$ of $s$-bounded functions is said to be uniformly $s$ bounded (or uniformly additive-cf. [3]) if for each $U \in \mathscr{U}$ and each sequence $\left\{E_{i}\right\}$ of pairwise disjoint elements of $\mathscr{S}$, there exists $m \in N$ such that $\sum_{i \in M} \mu_{k}\left(E_{i}\right) \in U$ whenever $k \in N$ and $M$ is a finite subset of $N^{m}$.

THEOREM 2. Suppose that $\left\{\mu_{k}\right\}$ is a sequence of $s$-bounded functions such that $\left\{\mu_{k}(E)\right\}$ is Cauchy for each $E \in \mathscr{S}$. Then $\left\{\mu_{k}\right\}$ is uniformly s-bounded.

ProOF. Suppose that $\left\{\mu_{k}\right\}$ is not uniformly s-bounded. Then there exists a sequence $\left\{E_{k}\right\}$ of pairwise disjoint elements of $\mathscr{S}, U \in \mathscr{U}$, a sequence $\left\{M_{k}\right\}$ of pairwise disjoint finite subsets of $N$, and an increasing sequence $\left\{n_{k}\right\}$ of elements of $N$ such that

(i) $\mu_{n_{k}}\left(F_{k}\right) \notin 5 U$, where $F_{k}=\bigcup_{i \in M_{k}} E_{i}$,

(ii) $b_{k}=\max \left\{i \in M_{k}\right\}<\min \left\{i \in M_{k+1}\right\}=a_{k+1}$,

(iii) if $M$ is a finite subset of $N_{b_{k-1}}=\left\{n \in N ; n \leqq b_{k-1}\right\}$, then $\left(\mu_{n_{k}}-\mu_{j}\right)\left(\bigcup_{i \in M} E_{i}\right) \in U, j>n_{k}$, and

(iv) if $M$ is a finite subset of $N^{a_{(k+1)}}$, then $\mu_{n_{k}}\left(\bigcup_{i \in M} E_{i}\right) \in U$.

Set $v_{k}=\mu_{n_{k}}-\mu_{n_{(k+1)}}$; then $v_{k}(E) \rightarrow 0, E \in \mathscr{S}$, and $\left\{F_{k}\right\}$ is a sequence of pairwise disjoint elements of $\mathscr{S}$ with $v_{k}\left(F_{k}\right) \notin 4 U, k \in N$. Partition $\left\{F_{i}\right\}_{i \geqq 2}$ into a sequence $\left\{\left\{F_{i_{j}}\right\}_{j \geqq 1}\right\}_{i \geqq 1}$ of subsequences. Since $v_{1}$ is $s$-bounded there 
is a least integer $i_{1}$ such that $i \geqq i_{1}$ implies that $\left\{v_{1}(E): E \in \mathscr{S}, E \subset\right.$ $\left.\bigcup_{j=1}^{\infty} F_{i j}\right\} \subset U$ (i.e., $\left.\left\{v_{1} \mid\left(\bigcup_{j \geqq 1} F_{i j}\right)\right\} \subset U\right)$. Then since $v_{i_{1}}$ is $s$-bounded we can repeat this process and choose a subsequence of $\left\{F_{\left(i_{1)}\right)}\right\}$ on which $v_{i_{1}}$ stays in $U$. This process may be iterated, after which a diagonalization and relabeling yields (cf. (iii)) $v_{k}\left(\bigcup_{i \in M \subset N_{k-1}} F_{i}\right) \in U, v_{k}\left(F_{k}\right) \notin 4 U$, and $v_{k}(F) \in U$ if $F \in \mathscr{S}$ and $F \subset\left(\bigcup_{j>k} F_{j}\right)$. Thus, the contradiction $v_{k}\left(\bigcup_{i \geqq 1} F_{i}\right)$ $\notin 2 U, k \in N$, obtains, and Theorem 2 is established.

An immediate corollary follows.

COROLlaRY 2. If $\left\{\mu_{k}\right\}$ is a sequence of s-bounded functions and $\mu(E)$ $=\lim \mu_{k}(E), E \in \mathscr{S}$, then $\mu$ is s-bounded.

In conclusion we remark that several generalizations are possible (cf. the theorems in [4]).

\section{REFERENCES}

1. R. B. Darst, The Lebesgue decomposition, Duke Math. J. 30 (1963), 553-556. MR 28 \#176.

2. - On a theorem of Nikodym with applications to weak convergence and von Neumann algebras, Pacific J. Math. 23 (1967), 473-477. MR 38 \#6360.

3. - The Vitali-Hahn-Saks and Nikodym theorems for additive set functions, Bull. Amer. Math. Soc. 76 (1970), 1297-1298. MR 41 \# 8625.

4. D. Landers and L. Rogge, The Vitali-Hahn-Saks and uniform boundedness theorem in topological groups, Manuscripta Math. 4 (1971), 351-359. MR 44 \# 402.

5. C. E. Rickart, Decomposition of additive set functions, Duke Math. J. 10 (1943), 653-665. MR 5, 232.

Department of Mathematics, Colorado State University, Fort Collins, Colorado 80521 